\section{TATRA \\ MOUNTaiNS \\ Mathematical Publications}

DOI: $10.2478 / \mathrm{tmmp}-2021-0018$

Tatra Mt. Math. Publ. 79 (2021), 33-46

\title{
TWO NON ALGEBRAIC LIMIT CYCLES OF A CLASS OF POLYNOMIAL DIFFERENTIAL SYSTEMS WITH NON-ELEMENTARY EQUILIBRIUM POINT
}

\author{
Sabah Benadouane $^{1}$ - Aziza Berbache ${ }^{2}$ - Ahmed Bendjeddou $^{1}$ \\ ${ }^{1}$ Department of Mathematics, University Ferhat Abbas Sétif 1, Sétif, ALGERIA \\ ${ }^{2}$ Department of Mathematics, University of Bordj Bou Arréridj, Bordj Bou Arréridj, ALGERIA
}

\begin{abstract}
The problems of existence of limit cycles and their numbers are the most difficult problems in the dynamical planar systems. In this paper, we study the limit cycles for a family of polynomial differential systems of degree $6 k+1, k \in \mathbb{N}^{*}$, with the non-elementary singular point. Under some suitable conditions, we show our system exhibiting two non algebraic or two algebraic limit cycles explicitly given. To illustrate our results we present some examples.
\end{abstract}

\section{Introduction}

One of the important problems in the qualitative theory of differential equations is to solve the second part of the 16th problem out of 23 problems that Hilbert presented at the international congress of mathematicians in Paris (1900), see [1]. Hilbert asked there for an upper bound for the maximum number of limit cycles of all polynomial differential systems of degree $n$ of the form

$$
\begin{aligned}
& \dot{x}=\frac{d x}{d t}=P(x, y), \\
& \dot{y}=\frac{d y}{d t}=Q(x, y),
\end{aligned}
$$

where $P(x, y)$ and $Q(x, y)$ are real polynomials in the variables $x$ and $y$.

(C) 2021 Mathematical Institute, Slovak Academy of Sciences.

2010 Mathematics Subject Classification: $34 \mathrm{~A} 05,34 \mathrm{C} 07,34 \mathrm{C} 25$.

Keywords: Algebraic and non-algebraic limit cycle, planar polynomial differential system, first integral.

The authors would like to express their thanks to the General Directory for Scientific Research and Technological Development (DGSRTD), MESRS Algeria and Research project under the code: PRFUNCOOLO3UN190120180007, for their financial supports.

(ㄷ) (1) $\Theta \Theta$ Licensed under the Creative Commons BY-NC-ND 4.0 International Public License. 


\section{SABAH BENADOUANE-AZIZA BERBACHE-AHMED BENDJEDDOU}

The degree of the system is the maximum of the degrees of the polynomials $P$ and $Q$.

System (11) is integrable on an open set $\Omega$ of $\mathbb{R}^{2}$ if there exists a non constant $C^{1}$ function $H: \Omega \rightarrow \mathbb{R}$, called a first integral of the system on $\Omega$, which is constant on the trajectories of the system (1) contained in $\Omega$, i.e., if

$$
\frac{d H(x, y)}{d t}=P(x, y) \frac{\partial H(x, y)}{\partial x}+Q(x, y) \frac{\partial H(x, y)}{\partial y} \equiv 0
$$

in the points of $\Omega$. Moreover, $H=h$ is the general solution of this equation, where $h$ is an arbitrary constant.

A function of the form $f_{1}^{\lambda_{1}} \ldots f_{p}^{\lambda_{p}} \exp \left(h / f_{0}\right)$, where $f_{i}$ and $h$ are polynomials in $\mathbb{C}[x, y]$ and the $\lambda_{i} \in \mathbb{C}$, is called a Darboux function, see for example [12, 13]. System (11) is called Darboux integrable if it has a first integral which is a Darboux function (for a definition of first integral see [7]). A Liouvillian function is a function which can be expressed by quadratures of elementary functions. For more details see [8].

It is well-known that for differential systems defined on the plane $\mathbb{R}^{2}$, the existence of a first integral determines their phase portrait, see [6, 9].

A limit cycle of system (11) is an isolated periodic solution in the set of all periodic solutions of system (1). If a limit cycle is contained in an algebraic curve of the plane then we say that it is algebraic, otherwise it is called non-algebraic. In general, it is not easy to distinguish when a limit cycle is algebraic or not.

For example, the limit cycle of the van der Pol differential system discovered in 1927 (see [15]) was not proved until 1995 by O d a n i [14 that it was non-algebraic. The van der Pol system can be written as a polynomial differential system (11) of degree 3 , but its limit cycle is not known explicitly.

In the last years, several papers were published exhibiting polynomial differential systems for which non-algebraic limit cycles are explicitly known. The first explicit non-algebraic limit cycle, due to G a s u ll, Gi a c o m in i and Torregros a [9], was for a polynomial differential system of degree 5. Afterwards, A l- D o s a ry [1] gave a family of polynomial differential systems, with an explicit non-algebraic limit cycle, which generalizes the system studied in [9]. $\mathrm{B}$ e n d j e d d o u in [3] provided a class of polynomial differential system of degree odd with explicit non-algebraic limit cycle.

The first result for the coexistence of algebraic and non-algebraic limit cycle goes back to J. Gin é and M. Gra u [10] for $n=9$. These authors transform their system into a Ricatti equation which is transformed into a variable coefficients second order linear differential equation using the classic linearization method. 
B endjed d o u et al. 2, 4, provide a polynomial differential system of degree 5 exhibiting simultaneously two explicit limit cycles one algebraic and another non-algebraic. In [5] a class of differential systems of degree $6 n+1$ with three explicit limit cycles which are not algebraic is presented.

In this paper, we are interested in studying the integrability and the limit cycles of systems with degenerate non-elementary singular point of the form

$$
\begin{aligned}
\dot{x}= & 2 x\left(x^{2}+y^{2}\right)^{k}+\left(\gamma-\left(x^{2}+y^{2}\right)^{k}\right)\left(2 c\left(x^{2}+y^{2}\right)^{k}-b P_{2 k}(x, y)\right) \\
& \times\left(a \gamma x-(a x-4 k y)\left(x^{2}+y^{2}\right)^{k}\right), \\
\dot{y}= & 2 y\left(x^{2}+y^{2}\right)^{k}+\left(\gamma-\left(x^{2}+y^{2}\right)^{k}\right)\left(2 c\left(x^{2}+y^{2}\right)^{k}-b P_{2 k}(x, y)\right) \\
& \times\left(a \gamma y-(a y+4 k x)\left(x^{2}+y^{2}\right)^{k}\right),
\end{aligned}
$$

where $a, b, c, \gamma \in \mathbb{R}, k$ is a positive integer $\left(k \in \mathbb{N}^{*}\right)$, and $P_{2 k}(x, y)$ is a homogeneous polynomial of degree $2 k$ such that

$$
P_{2 k}(x, y)=\sum_{s=0}^{k-1}(-1)^{s}\left(\begin{array}{c}
2 k \\
2 s+1
\end{array}\right) x^{2 k-2 s-1} y^{2 s+1},
$$

where

$$
\left(\begin{array}{c}
2 k \\
2 s+1
\end{array}\right)=\frac{2 k !}{(2 s+1) !(2 k-2 s-1) !} .
$$

Moreover, we determine sufficient conditions for a polynomial differential system to possess an explicit two non-algebraic or two algebraic limit cycles surrounding the origin point. Concrete examples exhibiting the applicability of our result are introduced.

We say that a singular point is non-elementary if both eigenvalues of the linear part of the vector field at that point are zero, and elementary otherwise. A nonelementary singular point is called degenerate if its linear part is identically zero, otherwise it is called nilpotent.

\section{Main result}

As a main result, we shall prove the following theorem.

Theorem 2.1. Consider polynomial differential system (2). Then the following three statements hold:

1. System (2) is Darboux integrable with the Liouvillian first integral

$$
I(x, y)=\left(\left(x^{2}+y^{2}\right)^{k}-\gamma\right)^{2} e^{-a \arctan \frac{y}{x}}-\int_{0}^{\arctan \frac{y}{x}} \frac{e^{-a s}}{c-\frac{1}{2} b \sin 2 k s} d s
$$


2. If $a<0, b \neq 0, c>\frac{1}{2}|b|, \gamma>0$ and $a \gamma^{2}\left(c-\frac{1}{2}|b|\right)+1<0$, then the system (2) has two explicit non-algebraic limit cycles, given in polar coordinates $(r, \theta)$ by

$$
\begin{aligned}
& r_{1}\left(\theta, r_{1}^{*}\right)=\left(\gamma+\sqrt{\varphi\left(\theta, r_{1}^{*}\right)}\right)^{\frac{1}{2 k}}, \\
& r_{2}\left(\theta, r_{2}^{*}\right)=\left(\gamma-\sqrt{\varphi\left(\theta, r_{2}^{*}\right)}\right)^{\frac{1}{2 k}},
\end{aligned}
$$

where

$$
\begin{gathered}
\varphi\left(\theta, r_{i}^{*}\right)=e^{a \theta}\left(\left(\left(r_{i}^{*}\right)^{2 k}-\gamma\right)^{2}+g(\theta)\right), \quad i=1,2, \\
g(\theta)=\int_{0}^{\theta} \frac{e^{-a s}}{c-\frac{1}{2} b \sin 2 k s} d s, \\
r_{1}^{*}=\left(\gamma+\sqrt{\frac{e^{2 \pi a}}{1-e^{2 \pi a}} g(2 \pi)}\right)^{\frac{1}{2 k}}, \\
r_{2}^{*}=\left(\gamma-\sqrt{\frac{e^{2 \pi a}}{1-e^{2 \pi a}} g(2 \pi)}\right)^{\frac{1}{2 k}} .
\end{gathered}
$$

3. If $b=0, a c<0, \gamma>0$ and $\gamma^{2}+\frac{1}{a c}>0$, then system (2) has two explicit algebraic limit cycles, given in Cartesian coordinates $(x, y)$ by

$$
\left(\left(x^{2}+y^{2}\right)^{k}-\gamma\right)^{2}+\frac{1}{a c}=0 .
$$

The following Lemma collects some results which we need to show the statements of Theorem 2.1.

Lemma 2.2. Let $a<0, c>\frac{1}{2}|b|, a \gamma^{2}\left(c-\frac{1}{2}|b|\right)+1<0$ and $\gamma>0$, then the following statements hold:

1. The function $\Phi(\theta)=\gamma^{2} e^{-a \theta}-g(\theta)$ is strictly increasing, for all $\theta \in[0,2 \pi[$, where $g(\theta)$ is a function defined in previous theorem.

2. $\frac{e^{2 \pi a}}{1-e^{2 \pi a}} g(2 \pi)<\gamma^{2} e^{-a \theta}-g(\theta)$.

3. $0<\varphi(\theta)<\gamma^{2}$, where $\varphi(\theta)=e^{a \theta}\left(\frac{e^{2 \pi a}}{1-e^{2 \pi a}} g(2 \pi)+g(\theta)\right)$. 
Proof of statement (1) of Lemma 2.2

We remark that the function $\Phi$ is differentiable for all $\theta \in[0,2 \pi[$, then

$$
\frac{d \Phi}{d \theta}=-\frac{e^{-a \theta}}{c-\frac{1}{2} b \sin 2 k \theta}\left(a \gamma^{2}\left(c-\frac{1}{2} b \sin 2 k \theta\right)+1\right) .
$$

Since $a<0, c>\frac{1}{2}|b|$ and $\gamma>0$, then

and

$$
0<c-\frac{1}{2}|b|<c-\frac{1}{2} b \sin 2 k \theta<\frac{1}{2}|b|+c
$$

$$
a \gamma^{2}\left(\frac{1}{2}|b|+c\right)+1<a \gamma^{2}\left(c-\frac{1}{2} b \sin 2 k \theta\right)+1<a \gamma^{2}\left(c-\frac{1}{2}|b|\right)+1 .
$$

Since $a \gamma^{2}\left(c-\frac{1}{2}|b|\right)+1<0$, then

$$
a \gamma^{2}\left(c-\frac{1}{2} b \sin 2 k \theta\right)+1<0, \text { hence } \Phi^{\prime}(\theta)>0 .
$$

Consequently, the function $\Phi$ is strictly increasing.

Proof of statement (2) of Lemma 2.2 .

Because $\Phi(\theta)$ is strictly increasing then we have $\Phi(0)<\Phi(\theta)<\Phi(2 \pi)$ equivalent to

$$
\gamma^{2}<\gamma^{2} e^{-a \theta}-g(\theta)<\gamma^{2} e^{-2 \pi a}-g(2 \pi) .
$$

We remark that $\gamma^{2}<\gamma^{2} e^{-2 \pi a}-g(2 \pi)$ which implies that

since $a<0$, then

$$
g(2 \pi)<\gamma^{2}\left(e^{-2 \pi a}-1\right)=\gamma^{2} \frac{\left(1-e^{2 \pi a}\right)}{e^{2 \pi a}},
$$

$$
\frac{e^{2 \pi a}}{1-e^{2 \pi a}} g(2 \pi)<\gamma^{2} .
$$

Taking into account (4) we obtain

$$
\frac{e^{2 \pi a}}{1-e^{2 \pi a}} g(2 \pi)<\gamma^{2} e^{-a \theta}-g(\theta) .
$$

Proof of statement (3) of Lemma 2.2.

First we prove that $\varphi(\theta)<\gamma^{2}$. From the statement 2 of Lemma 2.2, we have

$$
\begin{aligned}
\varphi(\theta) & =e^{a \theta}\left(\frac{e^{2 \pi a}}{1-e^{2 \pi a}} g(2 \pi)+g(\theta)\right) \\
& <e^{a \theta}\left(\gamma^{2} e^{-a \theta}-g(\theta)+g(\theta)\right) \\
& =\gamma^{2} .
\end{aligned}
$$


Then $\varphi(\theta)<\gamma^{2}$. Since $c>\frac{1}{2}|b|$ we get that $g(\theta)>0$ and we have $a>0$, it follows that $\frac{e^{2 \pi a}}{1-e^{2 \pi a}} g(2 \pi)>0$, hence $\varphi(\theta)>0$. Consequently,

$$
0<\varphi(\theta)<\gamma^{2}, \quad \text { for all } \theta \in[0 ; 2 \pi[.
$$

This completes the proof of Lemma 2.2

Proof of Theorem 2.1. First, we have

$$
y \dot{x}-x \dot{y}=4 k\left(\gamma-\left(x^{2}+y^{2}\right)^{k}\right)\left(2 c\left(x^{2}+y^{2}\right)^{k}-b P_{2 k}(x, y)\right)\left(x^{2}+y^{2}\right)^{k+1} .
$$

Thus, the equilibrium points of system (2) are presented in the equation curve's

$$
4 k\left(\gamma-\left(x^{2}+y^{2}\right)^{k}\right)\left(2 c\left(x^{2}+y^{2}\right)^{k}-b P_{2 k}(x, y)\right)\left(x^{2}+y^{2}\right)^{k+1}=0 .
$$

In polar coordinates $(r, \theta)$, where $x=r \cos \theta$ and $y=r \sin \theta$, the function $P_{2 k}(x, y)$ reads as

$$
\begin{aligned}
P_{2 k}(r \cos \theta, r \sin \theta) & =\sum_{s=0}^{k-1} C_{2 k}^{2 s+1}(-1)^{s}(r \cos \theta)^{2 k-2 s-1}(r \sin \theta)^{2 s+1} \\
& =r^{2 k} \sin (2 k \theta) .
\end{aligned}
$$

Then the curve's (6) can be written as

$$
4 k r^{2 k}\left(\gamma-r^{2 k}\right)(2 c-b \sin (2 k \theta)) r^{2 k+2}=0 .
$$

From the condition $c>\frac{1}{2}|b|$, we have $2 c-b \sin (2 k \theta)>0$, then the equilibrium points of system (2) are presented on the curve

$$
r^{2 k}\left(\gamma-r^{2 k}\right)=0
$$

We deduce that the origin is an equilibrium point which is a degenerate nonelementary singular point of system (2), because the linear part of this system is identically zero, and any other, if exists must lie on the curve

$$
\left(x^{2}+y^{2}\right)^{k}-\gamma=0 .
$$

To prove our results, we write the polynomial differential system (2) in polar coordinates $(r, \theta)$ defined by $x=r \cos \theta$ and $y=r \sin \theta$. Then the system becomes

$$
\begin{aligned}
& \dot{r}=r^{2 k+1}\left(2+a(2 c-b \sin 2 k \theta)\left(\gamma-r^{2 k}\right)^{2}\right), \\
& \dot{\theta}=-4 k(2 c-b \sin 2 k \theta)\left(\gamma-r^{2 k}\right) r^{4 k} .
\end{aligned}
$$

The differential system (요), where $4 k(2 c-b \sin 2 k \theta)\left(\gamma-r^{2 k}\right) r^{4 k} \neq 0$, can be written as the equivalent differential equation

$$
\frac{d r}{d \theta}=\frac{r\left(2+a(2 c-b \sin 2 k \theta)\left(\gamma-r^{2 k}\right)^{2}\right)}{-4 k(2 c-b \sin 2 k \theta)\left(\gamma-r^{2 k}\right) r^{2 k}} .
$$


Via the change of variables $\varphi=\left(r^{2 k}-\gamma\right)^{2}$, the equation (9) is transformed into the linear equation

$$
\frac{d \varphi}{d \theta}=a \varphi+\frac{1}{c-\frac{1}{2} b \sin 2 k \theta} .
$$

The general solution of this equation is

$$
\varphi(\theta, h)=e^{a \theta}(h+g(\theta)),
$$

where $h \in \mathbb{R}$ and

$$
g(\theta)=\int_{0}^{\theta} \frac{e^{-a s}}{c-\frac{1}{2} b \sin 2 k s} d s .
$$

Consequently, the implicit solution of equation (9) is given by

$$
\left(r^{2 k}-\gamma\right)^{2}=e^{a \theta}(h+g(\theta))
$$

By passing to Cartesian coordinates, we deduce that the first integral is

$$
I(x, y)=\left(\left(x^{2}+y^{2}\right)^{k}-\gamma\right)^{2} e^{-a \arctan \frac{y}{x}}-\int_{0}^{\arctan \frac{y}{x}} \frac{e^{-a s}}{c-\frac{1}{2} b \sin 2 k s} d s .
$$

Since this first integral is a function that can be expressed by quadratures of elementary functions, it is a Liouvillian function, and consequently system (2) is Darboux integrable.

Notice that system (2) has a periodic orbit if and only if the equation (9) has a strictly positive $2 \pi$-periodic solution. The solution satisfying the initial condition $r\left(0, r_{0}\right)=r_{0}>0$ is given by $h=\left(r_{0}^{2 k}-\gamma\right)^{2}$. Then, the implicit solution of equation (9) with this initial condition is

$$
\left(r^{2 k}-\gamma\right)^{2}=e^{a \theta}\left(\left(r_{0}^{2 k}-\gamma\right)^{2}+g(\theta)\right) .
$$

Since $\varphi=\left(r^{2 k}-\gamma\right)^{2}$, it is clear that

we have

$$
r\left(2 \pi, r_{0}\right)=r\left(0, r_{0}\right) \quad \text { if and only if } \varphi\left(2 \pi, r_{0}\right)=\varphi\left(0, r_{0}\right),
$$

$$
\varphi_{0}=\varphi\left(0, r_{0}\right)=\left(r_{0}^{2 k}-\gamma\right)^{2} \quad \text { and } \quad \varphi\left(2 \pi, r_{0}\right)=e^{2 \pi a}\left(\left(r_{0}^{2 k}-\gamma\right)^{2}+g(2 \pi)\right)
$$

Then, the condition $\varphi\left(2 \pi, r_{0}\right)=\varphi\left(0, r_{0}\right)$ implies that

Thus

$$
\left(r_{0}^{2 k}-\gamma\right)^{2}=\frac{e^{2 \pi a}}{1-e^{2 \pi a}} g(2 \pi)
$$

$$
\varphi(\theta)=e^{a \theta}\left(\frac{e^{2 \pi a}}{1-e^{2 \pi a}} g(2 \pi)+g(\theta)\right) .
$$




\section{SABAH BENADOUANE-AZIZA BERBACHE-AHMED BENDJEDDOU}

The implicit form of the solution of (9) can be written as

$$
\left(r^{2 k}-\gamma\right)^{2}=e^{a \theta}\left(\frac{e^{2 \pi a}}{1-e^{2 \pi a}} g(2 \pi)+g(\theta)\right) .
$$

From the expression of the change of variable $\varphi=\left(r^{2 k}-\gamma\right)^{2}$ that transform (9) into (10), one gets

$$
\begin{aligned}
& r_{1}(\theta)=(\gamma+\sqrt{\varphi(\theta)})^{\frac{1}{2 k}} \\
& r_{2}(\theta)=(\gamma-\sqrt{\varphi(\theta)})^{\frac{1}{2 k}}
\end{aligned}
$$

These two solutions are strictly positive because we have $\gamma>0$ and from the statement (3) of Lemma 2.2 we have $0<\varphi(\theta)<\gamma^{2}$.

From (13), there are two different values of $r_{0}$ with the property

$$
r\left(2 \pi ; r_{0}\right)=r_{0}>0
$$

given by

$$
\begin{aligned}
& r_{1}^{*}=\left(\gamma+\sqrt{\frac{e^{2 \pi a}}{1-e^{2 \pi a}} g(2 \pi)}\right)^{\frac{1}{2 k}}, \\
& r_{2}^{*}=\left(\gamma-\sqrt{\frac{e^{2 \pi a}}{1-e^{2 \pi a}} g(2 \pi)}\right)^{\frac{1}{2 k}} .
\end{aligned}
$$

Since $a<0, \gamma>0$ and by (5) we have $\frac{e^{2 \pi a}}{1-e^{2 \pi a}} g(2 \pi)<\gamma^{2}$, then $r_{1}^{*}>0$ and $r_{2}^{*}>0$. After the substitution of the values $r_{i}^{*}, i=1,2$ into $r_{i}(\theta), i=1,2$ we obtain

where

$$
\begin{aligned}
& r_{1}\left(\theta, r_{1}^{*}\right)=\left(\gamma+\sqrt{\varphi\left(\theta, r_{1}^{*}\right)}\right)^{\frac{1}{2 k}}, \\
& r_{2}\left(\theta, r_{2}^{*}\right)=\left(\gamma-\sqrt{\varphi\left(\theta, r_{2}^{*}\right)}\right)^{\frac{1}{2 k}},
\end{aligned}
$$

$$
\varphi\left(\theta, r_{i}^{*}\right)=e^{a \theta}\left(\left(\left(r_{i}^{*}\right)^{2 k}-\gamma\right)^{2}+g(\theta)\right), \quad i=1,2 .
$$

To show that $r_{i}(\theta), i=1,2$ are periodic solutions, we have to show that

a) there does not exist any singular point of (14),

b) the functions $\theta \longmapsto r_{i}(\theta), i=1,2$ are $2 \pi$-periodic,

c) $r_{i}(\theta)>0, i=1,2$ for all $\theta \in[0,2 \pi[$. 
a) We first prove that there is no singular point of (14). In particular, we shall prove that the curve (7) does not intersect the orbit (14). This curve in polar coordinates becomes $r^{2 k}-\gamma=0$. To show this, we have to show that the system

$$
\left\{\begin{aligned}
\left(r^{2 k}-\gamma\right)^{2}-e^{a \theta}\left(\frac{e^{2 \pi a}}{1-e^{2 \pi a}} g(2 \pi)+g(\theta)\right) & =0, \\
r^{2 k}-\gamma & =0 .
\end{aligned}\right.
$$

has no solutions.

From the second equation of system (16) we get that $r^{2 k}=\gamma$, we replace this value in the first equation, we obtain

$$
e^{a \theta}\left(\frac{e^{2 \pi a}}{1-e^{2 \pi a}} g(2 \pi)+g(\theta)\right)=0,
$$

which is a contradiction because

$$
e^{a \theta}\left(\frac{e^{2 \pi a}}{1-e^{2 \pi a}} g(2 \pi)+g(\theta)\right)=\varphi(\theta)
$$

and from Lemma 2.2 we have $0<\varphi(\theta)<\gamma^{2}$. So (16) has no solutions.

b) Periodicity. From (15) we say that $r_{i}\left(\theta, r_{i}^{*}\right), i=1,2$ are $2 \pi$-periodic if and only if $\varphi(\theta)$ is $2 \pi$-periodic, then we have

$$
\varphi(\theta+2 \pi)=e^{a(\theta+2 \pi)}\left(\frac{e^{2 \pi a}}{1-e^{2 \pi a}} g(2 \pi)+g(\theta+2 \pi)\right) .
$$

However,

$$
\begin{aligned}
g(\theta+2 \pi) & =\int_{0}^{\theta+2 \pi} \frac{e^{-a s}}{c-\frac{1}{2} b \sin 2 k s} d s \\
& =g(2 \pi)+\int_{2 \pi}^{\theta+2 \pi} \frac{e^{-a s}}{c-\frac{1}{2} b \sin 2 k s} d s .
\end{aligned}
$$

In the integral $\int_{2 \pi}^{\theta+2 \pi} \frac{e^{-a s}}{c-\frac{1}{2} b \sin 2 k s} d s$, we use the change of variable $w=s-2 \pi$, we obtain

$$
\begin{aligned}
g(\theta+2 \pi) & =g(2 \pi)+\int_{0}^{\theta} \frac{e^{-a(w+2 \pi)}}{c-\frac{1}{2} b \sin 2 k(w+2 \pi)} d w \\
& =g(2 \pi)+e^{-2 \pi a} g(\theta) .
\end{aligned}
$$

We replace

$$
g(\theta+2 \pi) \text { by } g(2 \pi)+e^{-2 \pi a} g(\theta) \text { in (17), }
$$

and after some calculations we obtain $\varphi(\theta+2 \pi)=\varphi(\theta)$, hence $\varphi(\theta)$ is $2 \pi$-periodic. Consequently, the functions $r_{i}\left(\theta, r_{i}^{*}\right), i=1,2$ are also $2 \pi$-periodic. 
c) Strict positivity of $r_{i}\left(\theta, r_{i}^{*}\right), i=1,2$, for all $\theta \in\left[0 ; 2 \pi\left[\right.\right.$. Since $0<\varphi(\theta)<\gamma^{2}$ and $\gamma>0$, we have $\gamma^{2}>\varphi(\theta)$, which implies $\gamma>\sqrt{\varphi(\theta)}$, hence

$$
\gamma-\sqrt{\varphi(\theta)}>0 \text { and } \gamma+\sqrt{\varphi(\theta)}>0
$$

Therefore $r_{i}\left(\theta, r_{i}^{*}\right), i=1,2$ are strictly positive.

In order to prove that the periodic orbit is hyperbolic limit cycle, we consider the equation (91), and we introduce the Poincaré return map

$$
\lambda \rightarrow \Pi(2 \pi, \lambda)=r(2 \pi, \lambda) .
$$

Therefore, periodic orbits of system (2) are hyperbolic limit cycles if and only if $\left.\frac{d r_{i}(2 \pi, \lambda)}{d \lambda}\right|_{\lambda=r_{i}^{*}} \neq 1, i=1,2$, where

$$
r_{1}^{*}=\left(\gamma+\sqrt{\frac{e^{2 \pi a}}{1-e^{2 \pi a}} g(2 \pi)}\right)^{\frac{1}{2 k}}, \quad r_{2}^{*}=\left(\gamma-\sqrt{\frac{e^{2 \pi a}}{1-e^{2 \pi a}} g(2 \pi)}\right)^{\frac{1}{2 k}}
$$

We have

$$
\begin{aligned}
& r_{1}(2 \pi, \lambda)=\left(\gamma+\sqrt{e^{2 a \pi}\left(\left(\lambda^{2 k}-\gamma\right)^{2}+g(2 \pi)\right)}\right)^{\frac{1}{2 k}}, \\
& r_{2}(2 \pi, \lambda)=\left(\gamma-\sqrt{e^{2 a \pi}\left(\left(\lambda^{2 k}-\gamma\right)^{2}+g(2 \pi)\right)}\right)^{\frac{1}{2 k}} .
\end{aligned}
$$

After some calculations we obtain

$$
\left.\frac{d r_{1}(2 \pi, \lambda)}{d \lambda}\right|_{\lambda=r_{1}^{*}}=\left.\frac{d r_{2}(2 \pi, \lambda)}{d \lambda}\right|_{\lambda=r_{2}^{*}}=e^{2 \pi a} \neq 1
$$

Consequently, the limit cycles of the differential equation (9) are hyperbolic (see for instance [16]).

Now we prove that these two limit cycles are not algebraic for $b \neq 0$. The curve defined by these two limit cycles is

$$
\left(r^{2 k}-\gamma\right)^{2}-e^{a \theta}\left(\frac{e^{2 \pi a}}{1-e^{2 \pi a}} g(2 \pi)+g(\theta)\right)=0 .
$$

More precisely, in Cartesian coordinates, $r^{2}=x^{2}+y^{2}, \theta=\arctan \frac{y}{x}$, this curve can be written as

$$
\begin{aligned}
G(x, y)=\left(\left(x^{2}+y^{2}\right)^{k}-\gamma\right)^{2} & \\
& -e^{a \arctan \frac{y}{x}}\left(\frac{e^{2 \pi a} g(2 \pi)}{1-e^{2 \pi a}}+\int_{0}^{\arctan \frac{y}{x}} \frac{e^{-a s}}{c-\frac{1}{2} b \sin 2 k s} d s\right)=0 .
\end{aligned}
$$


We remark that $G(x, y)=0$ is not a polynomial because there is no integer $n$ for which both $\frac{\partial^{n} G}{\partial x^{n}}$ and $\frac{\partial^{n} G}{\partial y^{n}}$ vanish identically, for example when calculating $\frac{\partial G}{\partial x}$ note that the expression $e^{a \arctan \frac{y}{x}}\left(\frac{e^{2 \pi a}}{1-e^{2 \pi a}} g(2 \pi)+\int_{0}^{\arctan \frac{y}{x}} \frac{e^{-a s}}{c-\frac{1}{2} b \sin 2 k s} d s\right)$ appear again, so for any order of derivation this expression will appear. Therefore the curve $G(x, y)=0$ is non-algebraic and the limit cycles of the system (2) will also be non-algebraic.

Proof of statement (3). If we take $b=0$, we get $g(\theta)=\frac{1}{a c}\left(1-e^{-a \theta}\right)$ and $g(2 \pi)=\frac{1}{a c}\left(1-e^{-2 \pi a}\right)$. Then

$$
\varphi(\theta)=\frac{-1}{a c} .
$$

Going back through the changes of variables $\left(r^{2 k}-\gamma\right)^{2}=\varphi$ and by passing to Cartesian coordinates $(x, y)$, we obtain

$$
\left(\left(x^{2}+y^{2}\right)^{k}-\gamma\right)^{2}+\frac{1}{a c}=0
$$

The system (2) has two algebraic limit cycles if and only if $a c<0, \gamma>\sqrt{-\frac{1}{a c}}$. This complete the proof of Theorem 2.1.

\section{Examples}

The following examples illustrate our result.

For $k=1$, we have

$$
P_{2}(x, y)=2 x y
$$

For $k=2$, we have

$$
\begin{aligned}
P_{4}(x, y) & =\left(\begin{array}{l}
4 \\
1
\end{array}\right) x^{3} y+(-1)\left(\begin{array}{l}
4 \\
3
\end{array}\right) x y^{3} \\
& =4 x^{3} y-4 x y^{3} .
\end{aligned}
$$

EXAMPLE. If we take $\gamma=2, c=2, b=1, a=-1$, then system (2) reads

$$
\begin{aligned}
\dot{x}= & 2 x\left(x^{2}+y^{2}\right)^{k}+\left(2-\left(x^{2}+y^{2}\right)^{k}\right)\left(4\left(x^{2}+y^{2}\right)^{k}-P_{2 k}(x, y)\right) \\
& \times\left(-2 x+(x+4 k y)\left(x^{2}+y^{2}\right)^{k}\right), \\
\dot{y}= & 2 y\left(x^{2}+y^{2}\right)^{k}+\left(2-\left(x^{2}+y^{2}\right)^{k}\right)\left(4\left(x^{2}+y^{2}\right)^{k}-P_{2 k}(x, y)\right) \\
& \times\left(-2 y-(4 k x-y)\left(x^{2}+y^{2}\right)^{k}\right) .
\end{aligned}
$$


This system has two non algebraic limit cycles whose expressions in polar coordinates $(r ; \theta)$ are

$$
\begin{aligned}
& r_{1}(\theta)=2+\sqrt{e^{-\theta}\left(\left(\left(r_{1}^{*}\right)^{2}-2\right)^{2 k}+g(\theta)\right)}, \\
& r_{2}(\theta)=2-\sqrt{e^{-\theta}\left(\left(\left(r_{2}^{*}\right)^{2 k}-2\right)^{2}+g(\theta)\right)},
\end{aligned}
$$

where

$$
g(\theta)=\int_{0}^{\theta} \frac{e^{s}}{2-\frac{1}{2} \sin 2 k s} d s
$$

$$
r_{1}^{*}=\left(2+\sqrt{\frac{e^{-2 \pi}}{1-e^{-2 \pi}} g(2 \pi)}\right)^{\frac{1}{2 k}}, \quad r_{2}^{*}=\left(2-\sqrt{\frac{e^{-2 \pi}}{1-e^{-2 \pi}} g(2 \pi)}\right)^{\frac{1}{2 k}} .
$$

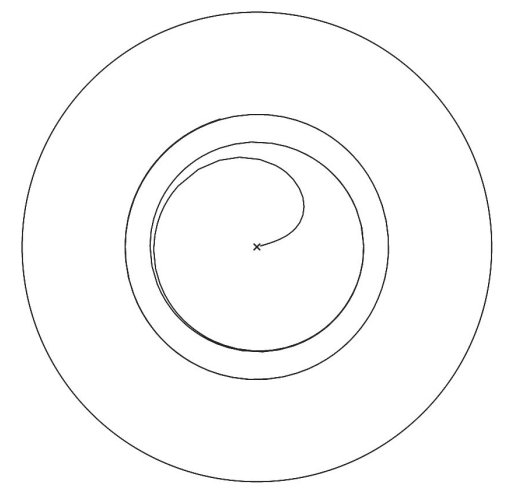

Case $\mathrm{k}=1$

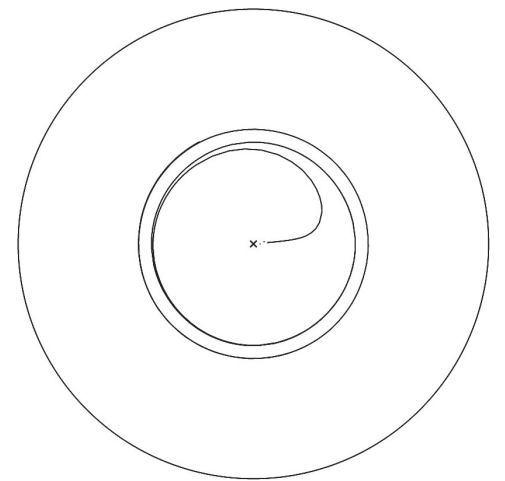

Case $\mathrm{k}=2$

Figure 1. Two non-algebraic limit cycles of system (20) in Poincaré disc.

ExAmple. Let $\gamma=2, c=2, b=0$ and $a=-1$, then the system (2) becomes

$$
\begin{gathered}
\dot{x}=2 x\left(x^{2}+y^{2}\right)^{k}-4\left(x^{2}+y^{2}\right)^{k}\left(2-\left(x^{2}+y^{2}\right)^{k}\right) \\
\times\left(2 x-(x+4 k y)\left(x^{2}+y^{2}\right)^{k}\right), \\
\dot{y}=2 y\left(x^{2}+y^{2}\right)^{k}-4\left(x^{2}+y^{2}\right)^{k}\left(2-\left(x^{2}+y^{2}\right)^{k}\right) \\
\times\left(2 y+(-y+4 k x)\left(x^{2}+y^{2}\right)^{k}\right) .
\end{gathered}
$$


We remark that this system satisfies the conditions of statement (3) of Theorem 2.1, hence the system (21) possesstwo algebraic limit cycles given in Cartesian coordinates by the expression

$$
\left(\left(x^{2}+y^{2}\right)^{k}-2\right)^{2}-\frac{1}{2}=0 .
$$

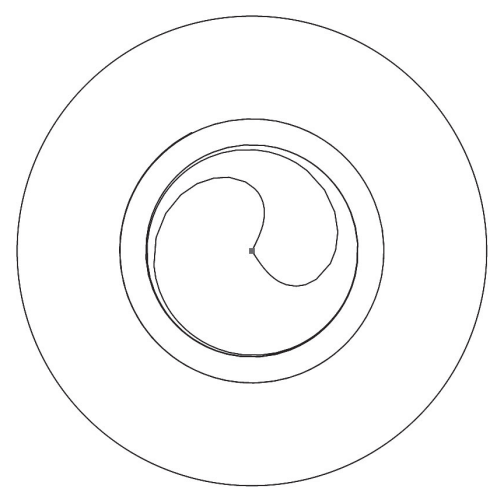

Case $\mathrm{k}=1$

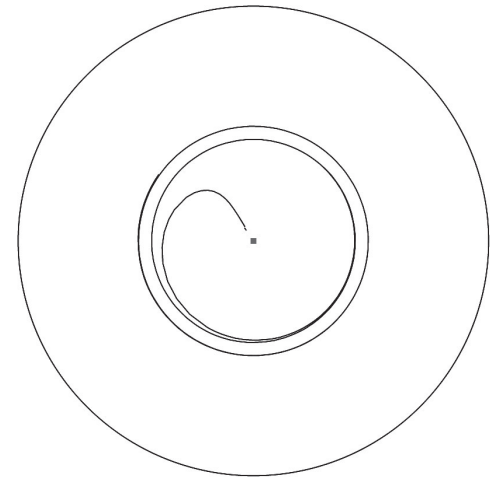

Case $\mathrm{k}=2$

Figure 2. Two algebraic limit cycles of system (21) in Poincaré disc.

\section{REFERENCES}

[1] AL-DOSARY, K. I T.: Non-algebraic limit cycles for parametrized planar polynomial systems, Internat. J. Math, 18 (2007), no. 2, 179-189.

[2] BENDJEDDOU, A.-BERBACHE, A.-CHEURFA, R.: Exact algebraic and non-algebraic limit cycles for a class of integrable quintic and planar polynomial differential systems, An. Ştiinţ. Univ. Al. I. Cuza Iaşi Mat. (N. S.) Tomul 64 (2018), no. 2. 253-259.

[3] BENDJEDDOU, A.-BERBACHE, A.: A class of differential system of odd degree with explicit non algebraic limit cycle, Int. Electron. J. Pure Appl. Math. 9 (2015), no.4, 243-254.

[4] BENDJEDDOU, A.-CHEURFA, R.: Coexistence of algebraic and non-algebraic limit cycles for quintic Polynomial differential systems, Electron. J. Differential Equations 2017, Paper no. 71, 7 pp.

[5] BERBACHE, A.-BENDJEDDOU, A.: Three explicit non-algebraic limit cycles for a class of polynomial differential systems, Appl. Math. E-Notes 20 (2020), 158-166.

[6] CAIRÓ, L.-LLIBRE, J.: Phase portraits of cubic polynomial vector fields of LotkaVolterra type having a rational first integral of egree 2, J. Phys. A 40 (2007), no. 24, 6329-6348.

[7] ChavarRiga, J.-GiaCOMini, H.- GINÉ, J.- LLIBRE, J.: Darboux integrability and the inverse integrating factor, J. Differential Equations 194 (2003), 116-139. 


\section{SABAH BENADOUANE-AZIZA BERBACHE-AHMED BENDJEDDOU}

[8] FERRAGUT, A.-LLIBRE, J.-MAHD, A.: Liouvillian first integrals of differential equations, Trans. Amer. Math. Soc. 333 (1992), no. 2, 673-688.

[9] GASUlL, A.-GIACOMINI, H.-TORREGROS, J.: Explicit non-algebraic limit cycles for polynomial systems, J. Comp. and Appl. Math. 200 (2007), 448-457.

[10] GINÉ, J.-GRAU, M.: Coexistence of algebraic and non-algebraic limit cycles, explicitly given, using Riccati equations, Nonlinearity 19 (2006), 1939-1950.

[11] HILBERT, D.: Mathematische Problem (lecture), In: Second International Congress Math. Paris, 1900, Nachr. Ges. Wiss. Gottingen Math-Phys. Kl. Vol. 1900, pp. 253-297.

[12] MOULIN, J. O.: About a conjecture on quadratic vector fields, J. Pure Appl. Algebra 165 (2001), 227-234.

[13] MOULIN, J. O.: Simple Darboux points of polynomial planar vector fields, J. Pure Appl. Algebra 189 (2004), 247-262.

[14] ODANI, K.: The limit cycle of the van der Pol equation is not algebraic, J. Differential Equations 115 (1995), 146-152.

[15] VAN DER POOL, B.: On relaxation-oscillations, Philos. Mag. VII. Ser. 2 (1926), 978-992.

[16] PERKO, L.: Differential Equations and Dynamical Systems (3rd edition). In: Texts in Appl. Math. Vol. 7, Springer-Verlag, New York, 2001.

Received November 13, 2020

\author{
Sabah Benadouane \\ Laboratory of Fundamental and \\ Numerical Mathematics \\ Department of Mathematics \\ Faculty of Sciences \\ University Ferhat Abbas Sétif 1 \\ Sétif-19000 \\ ALGERIA
}

E-mail: sabah.benadouane@univ-setif.dz

Aziza Berbache

Laboratory of Applied Mathematics

University Ferhat Abbas Sétif 1

Department of Mathematics

Faculty of Mathematics and Informatics

University of Bordj Bou Arréridj

Bordj Bou Arréridj-34265

ALGERIA

E-mail: azizaberbache@hotmail.fr

Ahmed Bendjeddou

Laboratory of Applied Mathematics

Department of Mathematics

Faculty of Sciences

University Ferhat Abbas Sétif 1

Sétif-19000

ALGERIA

E-mail: bendjeddou@univ-setif.dz 